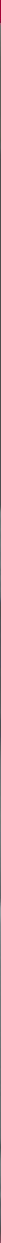

Unfallforschung kompakt

\title{
Praktische Führerscheinausbildung - ein Beitrag zur Optimierung
}

Unfallforschung
der Versicherer

$\neg$ GDV 


\section{Impressum}

Gesamtverband der Deutschen Versicherungswirtschaft e. V.

Unfallforschung der Versicherer

Wilhelmstraße 43/43G, 10117 Berlin

Postfach 0802 64, 10002 Berlin

unfallforschung@gdv.de

www.udv.de

Redaktion: Petra Butterwegge

Layout: Franziska Gerson Pereira

Bildnachweis: Unfallforschung der Versicherer

Erschienen: 09/2011 


\section{Vorbemerkung}

Junge Fahranfänger sind nach wie vor die herausragende Risikogruppe im Unfallgeschehen auf deutschen Straßen.

Im Jahr 2010 verunglückten nach Angaben des Statistischen Bundesamtes insgesamt 3.648 Verkehrsteilnehmer tödlich, 690 davon im Alter von 18 bis 24 Jahren. Der Bevölkerungsanteil dieser Gruppe beträgt jedoch gerade einmal $8 \%$, ihr Anteil am Unfallgeschehen hingegen fast $20 \%$. Das Risiko zu verunglücken ist dem- nach für Angehörige dieser Altersgruppe deutlich erhöht.

Auch wenn sich in den vergangenen Jahren ein positiver Trend erkennen lässt, müssen weitere Anstrengungen zur Erhöhung der Verkehrssicherheit junger Fahranfänger unternommen werden. Die vorliegende Studie möchte hierzu einen Beitrag leisten, indem sie die obligatorische praktische Fahrausbildung beleuchtet und mögliche Optimierungsansätze formuliert. 


\section{Inhalt}

Vorbemerkung

$1 \quad$ Einleitung

4

2 Unfallgeschehen

4

$3 \quad$ Struktur der Fahrausbildung

5

4 Diskussion

$5 \quad$ Ansätze zur Optimierung

$6 \quad$ Fazit

Literatur 


\section{$1 \quad$ Einleitung}

Das Unfallgeschehen junger Fahranfänger weist in den ersten Jahren der selbstständigen Verkehrsteilnahme deutlich erhöhte Risiken auf. So wurden 2010 [1] über $70 \%$ der Unfälle, an denen 18 bis 20-jährige Pkw-Fahrer beteiligt waren, auch von diesen verursacht. Mit steigendem Lebensalter sinkt dieser Anteil. In der Altersgruppe der 35 bis 55-Jährigen liegt er mit etwa $49 \%$ am niedrigsten.

Unfallforscher verschiedener Fachdisziplinen sind sich einig, dass das Fahrverhalten von Fahranfängern durch die Unerfahrenheit als Pkw-Führer (Anfängerrisiko), aber auch durch ein altersbedingtes Risikoverhalten (Jugendlichkeitsrisiko) geprägt ist. Erschwerend kommt hinzu, dass selbst einfache Fahraufgaben wie z.B. Kuppeln, Blinken, Blicke in den Spiegel ohne das Umfeld dabei aus den Augen zu verlieren, noch kaum automatisiert sind. Sie erfordern vom Fahranfänger eine bewusste Fokussierung auf die Aufgabe, was zu Defiziten in der Bewältigung der gesamten Verkehrssituation führen kann.

Die Unfallforschung der Versicherer (UDV) hat die Gefährdung junger Fahranfänger zum Anlass genommen, eine Studie aufzulegen, die die typischen Unfallausprägungen junger Fahrer aufzeigt, sie mit Bezug zur praktischen Fahrausbildung in Fahrschulen bewertet und Optimierungsansätze diskutiert.

Grundlage der Analyse des Unfallgeschehens waren Daten des Statistischen Bundesamtes (Destatis) aus den Jahren 2007 und 2008 für 18 und 19-jährige Pkw-Fahranfänger, sowie Auswertungen der UDV-eigenen Unfalldatenbank (UDB) für die Kalenderjahre 2002 bis 2005. Es wurden außerdem die relevanten Rechtsgrundlagen zur Fahrlehrer- und Fahrschülerausbildung unter besonderer Berücksichtigung der praktischen Fahrausbildung und Lehrunterlagen ohne Rechtsbindung begutachtet.

Die Ergebnisse der Forschungsarbeit [2] sind im Folgenden zusammenfassend dargestellt.

\section{Unfallgeschehen}

Die Unfalldatenbank der Versicherer ist eine sogenannte In-Depth-Datenbank. Derartige Datenbanken sind charakterisiert durch weniger umfangreiche Fallzahlen (für die vorliegende Studie $n=144$ ), aber eine große Informationstiefe je Unfall. Die Daten von Destatis hingegen zeichnen sich durch die hohen Fallzahlen (für diese Untersuchung $n=104$.243) aus, allerdings mit deutlich geringerer Informationstiefe für den Einzelfall. Die Analyse beider Datenquellen ermöglicht umfassende Erkenntnisse zu den Unfallausprägungen der Fahranfänger.

Nachfolgend werden ausgewählte Unfallstrukturen aufgezeigt, bei denen sich junge und erfahrene Fahrer (25 - 54 Jahre) unterscheiden:

- Junge Fahrer verunglücken zu gleichen Teilen innerhalb wie auch außerhalb von geschlossenen Ortschaften, erfahrene Fahrer mehrheitlich innerorts (ca. 60\%).

- Für 18-jährige Fahranfänger dominieren Unfälle, bei denen der Fahrer die Kontrolle über sein Fahrzeug verliert und dann von der Fahrbahn abkommt, ohne einen direkten Einfluss anderer Verkehrsteilnehmer (Fahrunfälle). Es folgen Auffahr- und Überholunfälle (Unfälle im Längsverkehr). Diese Reihung stellt sich bei erfahrenen Fahrern um.

- Junge Fahrer verunglücken häufiger als erfahrene Fahrer am Wochenende (25\% vs. 14\%).

- Führerscheinneulinge sind öfter an Unfällen bei Dunkelheit beteiligt (22\% vs. 12\%).

- Die sogenannten Disko-Unfälle sind häufig eine Kombination aus Wochenendfahrten bei Dunkelheit. 
Aus den Tiefenanalysen der UDB lassen sich weitere Hinweise ableiten:

- Fahranfänger sind selten allein unterwegs, im Mittel sind die Fahrzeuge mit zwei gleichaltrigen Mitfahrenden besetzt.

- Die meisten Unfälle ereignen sich auf Fahrten zu Orten, die für Jugendliche typisch sind wie Arbeits-/ Ausbildungsstätte, Gaststätten, andere Freizeiteinrichtungen.

- Der Anteil der 18-jährigen Führerscheinneulinge am Unfallaufkommen ihrer Altersgruppe ist größer als bei den 19-jährigen Fahranfängern.

- Die Pkw sind im Mittel acht bis neun Jahre alt und verfügen über eine Motorleistung zwischen 40 bis $70 \mathrm{~kW}$.

- Die Gurtanlegequote für Fahrer und Beifahrer liegt bei nahezu $100 \%$, für die hinteren Insassen zeigen sich geringere Quoten.

- Als ablenkende Tätigkeiten während des Fahrens konnten den Unfallakten Situationen wie "Spiegel einstellen, CD wechseln“ entnommen werden.

\section{$3 \quad$ Struktur der Fahrausbildung}

In Deutschland muss zum Erwerb der Fahrerlaubnis eine Fahrschule besucht werden. Die Führerscheinanwärter erhalten dort eine theoretische und praktische Ausbildung, die sie nach bestandener Prüfung befähigen soll, sicher am Straßenverkehr teilzunehmen. Es ist Aufgabe der Fahrlehrer, diese Befähigung zu vermitteln. Der Fokus der vorliegenden UDVStudie lag auf dem praktischen Teil der Ausbildung.

\section{Gesetzliche Grundlagen}

Zum besseren Verständnis wird im Folgenden umrissen, welche Vorgaben und Ziele der Gesetzgeber hierzu für die Ausbildungskräfte festgelegt hat.
Den Rahmen für die Fahrschulausbildung gibt das Straßenverkehrsgesetz (StVG) vor, so z. B. in $\S 2$ StVG zur grundsätzlichen Fahreignung.

Deutlich konkreter wird das Fahrlehrergesetz (FahrlG), in dem u. a. die zentralen Pflichten der Fahrlehrer benannt werden; professionelle und gewissenhafte Ausbildung der Fahrschüler: „Er hat ihnen die Kenntnisse, Fähigkeiten und Verhaltensweisen zu vermitteln, die das StraBenverkehrsgesetz und die auf diesem sowie auf dem Fahrlehrergesetz beruhenden Rechtsverordnungen für die Ausbildung und Prüfung der Bewerber um die Erlaubnis zum Führen von Kraftfahrzeugen fordern. Ferner hat er sie über die Folgen von Zuwiderhandlungen gegen die Verkehrsvorschriften .... zu unterrichten."

Auch die Eingangsvoraussetzungen zum Ergreifen des Berufes „Fahrlehrer" sowie die Bestimmungen zur Fahrlehrerausbildung sind im Fahrlehrergesetz festgeschrieben. Grundsätzliche Voraussetzungen sind z. B. ein Mindestalter von 22 Jahren, der Hauptschulabschluss und eine erfolgreich abgeschlossene Ausbildung in einem anerkannten Lehrberuf sowie eine Fahrpraxis von drei Jahren für die Fahrerlaubnisklasse B. Die Ausbildung selbst umfasst eine fünfmonatige Grundausbildung und ein viereinhalbmonatiges Praktikum in einer anerkannten Ausbildungsfahrschule. Die abzulegenden Prüfungen für beide Module sind in der Prüfungsordnung für Fahrlehrer (FahrlPrüfO) festgelegt und beschrieben. Alle vier Jahre müssen Fahrlehrer eine dreitägige Fortbildung absolvieren. Die Überwachung der Fahrlehrer, Fahrschulen und Fahrlehrerausbildungsstätten obliegt der örtlich zuständigen Fahrerlaubnisbehörde.

Die bedeutendste Rechtsgrundlage für den praktizierenden Fahrlehrer ist die FahrschülerAusbildungsverordnung (FahrschAusbO). Sie konkretisiert die übergeordneten Ziele der 
Fahrausbildung und definiert die Ausbildungsinhalte für Theorie und Praxis. Dort heißt es z. B. „Ziel der Ausbildung ist die Befähigung zum sicheren, verantwortungsvollen und umweltbewussten Verkehrsteilnehmer. Ziel der Ausbildung ist außerdem die Vorbereitung auf die Fahrerlaubnisprüfung." Zur praktischen Fahrausbildung wird in $\S 5$ ausgeführt: „Der praktische Unterricht ist auf die theoretische Ausbildung zu beziehen und inhaltlich mit dieser zu verzahnen. Er hat sich an den in den Anlagen 3 bis 6 aufgeführten Inhalten zu orientieren und die praktische Anwendung der Kenntnisse einzubeziehen, die zur Beurteilung der Verkehrs- und Betriebssicherheit des Fahrzeuges erforderlich sind. Er ist systematisch aufzubauen. Der praktische Unterricht besteht aus einer Grundausbildung und besonderen Ausbildungsfahrten...". Fahrschüler sollen also das Kraftfahrzeug bedienen lernen und soziale Kompetenz im Straßenverkehr vermittelt bekommen.

Fasst man die Anforderungen an Fahrlehrer bezüglich der Ausführungen in den Gesetzen zusammen, so muss jeder Ausbilder

- sowohl über pädagogische als auch didaktische Fähigkeiten verfügen,

- technisches Verständnis und verkehrsrechtliches Wissen aufweisen,

- Situationen reflektieren und professionelles Zeitmanagement umsetzen können.

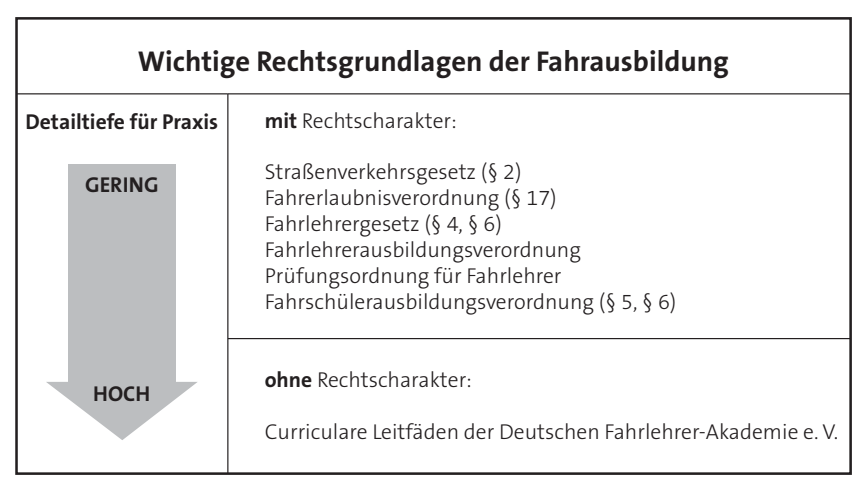

Abbildung 1:

Übersicht der wichtigsten Rechtsgrundlagen und ihre Informationstiefe für die Umsetzung in die Praxis
- Betreibt er eine Fahrschule, muss der Fahrlehrer auch kaufmännisches Geschick zeigen.

\section{Praxis}

In der beruflichen Praxis ist die Fahrschüler-Ausbildungsverordnung die wichtigste Grundlage für die Gestaltung der Ausbildung. Die Deutsche Fahrlehrer-Akademie e.V. gibt zur Unterstützung „Curriculare Leitfäden“ heraus. Sie besitzen keinen Rechtscharakter, sondern sind als Ergänzung „von Fahrlehrern für Fahrlehrer"zur Fahrschüler-Ausbildungsverordnung zu verstehen. Als ein wesentliches Ziel der Leitfäden wird die Hilfe „bei der Auseinandersetzung zwischen pädagogischem Auftrag und kaufmännischem Erwerbsstreben" formuliert. Aber auch die Diskussion zur Problematik des Jugendlichkeitsrisikos wird geführt und im Zusammenhang mit den Erläuterungen von Fahrübungen immer wieder thematisiert und es werden Tipps zum pädagogischen Vorgehen gegeben.

Fahrschüler erhalten nach Fahrschüler-Ausbildungsverordnung eine Grundausbildung und im Anschluss daran sogenannte besondere Ausbildungsfahrten. Zur praktischen Fahrausbildung "gehören auch die Anleitung .... und Hinweise vor, während und nach der Durchführung der Fahraufgaben sowie eine Nachbesprechung und Erörterung des jeweiligen Ausbildungsstandes." In der FahrschülerAusbildungsverordnung heißt es weiter: „Die gleichzeitige Erteilung von praktischem Fahrunterricht für mehrere Fahrschüler ist unzulässig."

Für die Grundausbildung bestehen keine zeitlichen oder örtlichen Vorgaben, es liegt im Ermessen des Ausbilders, wann sie als abgeschlossen gilt. Erlernt werden grundlegende Fahraufgaben zur Fahrgeschwindigkeit, zur Annäherung an Knotenpunkte u.a. In der 
Fahrschüler-Ausbildungsverordnung werden in Anhang 3 insgesamt 57 "Sachgebiete“ in Schlagwortcharakter aufgezählt, eine genauere Beschreibung erfolgt nicht. Die Curricularen Leitfäden hingegen beschreiben für jede Übungseinheit die Lerninhalte und Lernziele und geben Hinweise zur Didaktik und Methodik. Eine Fahrstunde umfasst, ähnlich einer Schulstunde, 45 Minuten.

Für die besonderen Ausbildungsfahrten nach Anhang 4 der Fahrschüler-Ausbildungsverordnung - in den Leitfäden Sonderfahrten genannt - werden Mindestvorgaben für Dauer und Häufigkeit gemacht. Die für das Unfallgeschehen der Fahranfänger besonders relevanten Fahrten sind:

- Überlandfahrt: 5 Fahrstunden auf Landstraßen, davon eine Fahrt von mindestens 90 Minuten,

- Fahrten bei Dämmerung/Dunkelheit: 3 Fahrstunden, davon je $50 \%$ auf Landstraßen bzw. Autobahnen. Diese Fahrten sind zusätzlich zu den anderen Sonderfahrten durchzuführen.

Unter Berücksichtigung der ebenfalls zu absolvierenden Fahrten auf Autobahnen / Kraftfahrstraßen (mindestens 4 Ausbildungsstunden, davon mindestens eine Fahrt von 90 Minuten) übt der Fahrschüler auf dem außerörtlichen Straßennetz also nur etwa 9 Stunden tatsächlich im Realververkehr.

Für den innerörtlichen Bereich werden keine besonderen Ausbildungsfahrten definiert. Darf man den Foren im Internet glauben, so benötigen die meisten jungen Fahrschüler 10 bis 20 Fahrstunden exklusive der besonderen Ausbildungsfahrten. Damit kommt der durchschnittliche Fahrschüler auf insgesamt circa 16 bis 24 Stunden, die er mit einem Fahrlehrer auf der Straße verbringt.

\section{Diskussion}

Die nachfolgende Diskussion interpretiert die vorangestellten Ausführungen und formuliert bedeutsame Aspekte der praktischen Fahrausbildung, für die in Kapitel 5 Optimierungsansätze vorgestellt werden. Dabei sollen nicht die besonders risikobereiten Fahranfänger im Zentrum der Betrachtungen stehen, sondern alle jungen Fahrer. Nicht alle 690 der tödlich Verunglückten zwischen 18 und 24 Jahren im Jahr 2010 waren „Rowdies“, aber alle waren unerfahren und jung.

\section{(1) Anfängerrisiko}

Die klassische Fahrausbildung ab 18 Jahren vermittelt Grundlagen für das Handling eines Pkw, die Inhalte der Straßenverkehrs-Ordnung für regelgerechtes Verhalten, macht den Fahrschüler bekannt mit möglichen Situationen im Verkehr und übt das selbstständige Führen eines Autos in den Fahrstunden. Der Übungsumfang hängt ganz wesentlich von den Fortschritten des Anwärters ab. Es ist davon auszugehen, dass der durchschnittliche Fahrschüler sich etwa zwischen 16 bis 24 Stunden im Verkehr bewegt. Er wird aber sicher nicht die gesamte Zeit am fließenden Verkehr teilnehmen, da z.B. Einpark-Übungen viel Zeit, aber wenig gefahrene Kilometer bedeuten. Erfahrungsaufbau braucht aber Zeit und Fahrleistung um vom Anfänger zum Routinier zu werden.

Die heutige Fahrschulausbildung kann also nur als ein erster Schritt zum sicheren Fahrer gewertet werden, Erfahrungsaufbau erfolgt erst nach bestandener Fahrerlaubnisprüfung.

\section{(2) Jugendlichkeitsrisiko}

Jugendliche im Alter von 18 und 19 Jahren befinden sich immer noch in der Pubertätsphase. Sie behindert souveränes Verhalten z. B. durch 
Ablösungs- oder Selbstfindungsprozesse. Die Fähigkeit, sich selbst richtig einzuschätzen, ist deutlich eingeschränkt. Vor allem männliche Jugendliche sind der Auffassung alles zu können, alles zu wissen und "unverwundbar" zu sein, die Welt des Jugendlichen dreht sich vorrangig um inn selbst. In der Entwicklungspsychologie werden diese Verhaltensmuster als "Invincibility-fable“ Phänomen und ,jugendlicher Egozentrismus" bezeichnet.

Pädagogisches und psychologisches Können ist eine wesentliche Voraussetzung um Jugendliche für Werte, wie sicheres oder defensives Fahren, zu motivieren. Daher sind sowohl die Eingangsvoraussetzungen für die Ausbildung zum Fahrlehrer, als auch die Dauer von nur neuneinhalb Monaten kritisch zu sehen.

\section{(3) Gruppendynamik}

Der Führerscheinneuling ist häufig mit Insassen seiner Altersstufe unterwegs. Auf engstem Raum in einem Pkw kann sich schnell eine Gruppendynamik aufbauen, die den Fahrzeugführer unter Druck setzt. Fordert die Peergroup vehement und massiv z.B. zu schnellerem Fahren auf, fehlt dem Fahrzeugführer sicher zu oft das Selbstbewusstsein sich dagegen zur Wehr zu setzten.

Diese bedeutende Thematik kann vom Fahrlehrer aber nur in der Theorie behandelt werden, da es nach Fahrschüler-Ausbildungsverordnung nicht erlaubt ist, praktischen Fahrunterricht für mehrere Anwärter zu erteilen und eine derartige Situation nur schwer real umsetzbar ist. Außerdem werden sich Fahrschüler im Beisein eines Fahrlehrers zurückhaltender verhalten.

\section{(4) Realitätsbezug}

Die Fahrstunden können nur einen Ausschnitt von Verkehrssituation lehren und üben. Insbe- sondere kritische Situationen, die im Realverkehr nicht ohne Gefährdung von Fahrlehrer und Fahrschüler erlebt werden können, z.B. eine Gefahrenvollbremsung oder Kurvenfahrten mit unangepasster Geschwindigkeit, können demnach nur in der Theorie vermittelt werden. Ähnliches gilt auch für Fahranwärter, die in den Sommermonaten ihren Führerschein machen: Angemessenes Fahrverhalten bei Eis und Schnee kann nur theoretisch behandelt werden.

An diesem Punkt sind auch dem motiviertesten Fahrlehrer Grenzen gesetzt. Er hat nur die Möglichkeit, die Situationen, die er vorfindet, so gut es geht zu nutzen und gegebenenfalls in der Nachbesprechung vertiefende Hinweise für sicheres Fahren zu geben.

\section{$5 \quad$ Ansätze zur Optimierung}

Vor etwa zehn Jahren wurden die Ergebnisse der europäischen GADGET-Studie [3] vorgestellt. Eines der wichtigsten Ergebnisse war dabei die Definition der GDE-Matrix (Goals for Driver Education) [4]. In vier hierarchisch angeordneten Ebenen formuliert sie die Ziele der Fahrausbildung. Heute ist die GDE-Matrix Stand der Wissenschaft innerhalb der EU und auch für die deutsche Fahrausbildung anerkannter Standard.

Die vier Ebenen der GDE-Matrix sind:

- Persönliche Werthaltungen und Einstellungen, Lebensziele usw. (höchste Ebene)

- Fahrmotive, Fahrtzweck, Fahrtumstände

- Bewältigung unterschiedlichster Verkehrssituationen

- Fahrzeugbedienung (niedrigste Ebene).

Obwohl die Matrix in Hierarchien aufgesetzt ist, greifen die Ebenen dennoch ineinander. So kann z. B. der jugendliche Fahranfänger trotz Defizite in den unteren Ebenen der Fahrzeug- 


\begin{tabular}{|c|c|c|c|}
\hline \multicolumn{4}{|c|}{$\begin{array}{c}\text { GDE-Matix } \\
\text { (Hatakka, Keskinen, Glad, Gregersen, Hernetkoski; 2002) }\end{array}$} \\
\hline & Wissen und Können & Risikoerhöhende Faktoren & Selbstbeurteilung \\
\hline $\begin{array}{l}\text { Persönliche } \\
\text { Werthaltungen und } \\
\text { Einstellungen, } \\
\text { Lebensziele usw. }\end{array}$ & $\begin{array}{l}\text { Lebensstil, Alter, Gruppe, } \\
\text { Kultur, soziale Stellung } \\
\text { usw. gegenüber dem } \\
\text { Fahrverhalten }\end{array}$ & $\begin{array}{c}\text { Abenteuerlust, } \\
\text { Risikobereitschaft, } \\
\text { Gruppendruck, geringere } \\
\text { Verantwortungsbereitschaft }\end{array}$ & $\begin{array}{l}\text { Fähigkeit zur Intro- } \\
\text { spektion - } \\
\text { Was bin ich für } \\
\text { ein Mensch? }\end{array}$ \\
\hline $\begin{array}{c}\text { Fahrmotive, } \\
\text { Fahrtzweck } \\
\text { und Fahrtumstände }\end{array}$ & $\begin{array}{c}\text { Wahl des Transportmittels, } \\
\text { Wahl der Zeit, } \\
\text { Rolle von Motiven, } \\
\text { Streckenplanung }\end{array}$ & $\begin{array}{l}\text { Unaufmerksamkeit, Alkohol, } \\
\text { Müdigkeit, Stoßzeiten, } \\
\text { junge Beifahrer, } \\
\text { Geschwindigkeitsrausch }\end{array}$ & $\begin{array}{l}\text { Selbstkontrolle, nicht } \\
\text { durch falsche eigene } \\
\text { Motive beeinflussen } \\
\text { lassen }\end{array}$ \\
\hline Fahren im Verkehr & $\begin{array}{c}\text { Verkehrsregeln, } \\
\text { Partnerkunde, } \\
\text { Wahrnehmen von } \\
\text { Gefahren, } \\
\text { Automatisierung }\end{array}$ & $\begin{array}{l}\text { Missachtung von Regeln, } \\
\text { zu dichtes Auffahren, } \\
\text { Glätte, } \\
\text { schwächere } \\
\text { Verkehrsteilnehmer }\end{array}$ & $\begin{array}{c}\text { Angepasstheit der } \\
\text { fahrerischen Fähigkeiten, } \\
\text { eigener Fahrstil }\end{array}$ \\
\hline $\begin{array}{l}\text { Fahrzeugbedienung, } \\
\text { Kontrolle über das } \\
\text { Fahrzeug }\end{array}$ & $\begin{array}{l}\text { Funktionen des Fahrzeugs, } \\
\text { Sicherheitssysteme, } \\
\text { Kontrolle über das } \\
\text { Fahrzeug, } \\
\text { physikalische Gesetze }\end{array}$ & $\begin{array}{l}\text { ohne Sicherheitsgurt, } \\
\text { Versagen der } \\
\text { Fahrzeugsysteme, } \\
\text { abgefahrene Reifen, } \\
\text { ungeschickt }\end{array}$ & $\begin{array}{l}\text { Angepasstheit der } \\
\text { Fähigkeit zur Kontrolle } \\
\text { des Fahrzeugs }\end{array}$ \\
\hline
\end{tabular}

\section{Abbildung 2:}

\section{GDE-Matrix mit den wesentlichen Ausbildungsinhalten der vier Ebenen}

bedienung und Verkehrssituationen sicher unterwegs sein, wenn es ihm gelingt, sein fahrerisches Können richtig einzuschätzen und er sich risikoerhöhenden Faktoren wie Gruppendruck widersetzt oder gar nicht erst aussetzt. Fahrausbildung im Sinn der GDEMatrix ist demnach nicht nur die Vermittlung von Wissen, sondern beinhaltet auch die Anleitung junger Menschen zu sozialen Werten und Verhaltensweisen.

Ausgehend von der Zielsetzung der GDEMatrix, den Erkenntnisse der vorliegenden Untersuchung und bereits evaluierten (Pilot-) Projekten werden folgende bedeutsame Optimierungspotenziale gesehen:

\section{(1) Mehrphasen-Fahrausbildung}

Fahranfänger brauchen die Chance, Fahrkompetenz aufzubauen, ohne sich dabei zu großen Gefahren aussetzen zu müssen. Es gilt, für sie Fahrerfahrung unter geschützten Bedingungen zu entwickeln. Wichtiger Faktor hierfür sind die selbstständig gefahrenen Kilometer.

- Der Modellversuch „Begleitetes Fahren ab 17 (BF 17)" hat sich in diesem Zusammenhang bewährt und ist inzwischen geltendes Recht. BF 17 sollte weiter beworben werden, um die Teilnahmequote von derzeit etwa $35 \%$ noch zu erhöhen.

- Nicht alle Führerscheinanwärter haben die Möglichkeit an BF17 teilzunehmen. Daraus ergibt sich die Notwendigkeit, eine Ausbildungsstruktur aufzubauen, die alle Bewerber erreicht. Hier sollte von den Nachbarländern Österreich und Schweiz partizipiert werden. Es gilt ein auf Deutschland passendes Ausbildungsmodell zu entwickeln, in dem die Fahrschulausbildung in mehreren Phasen gegliedert wird und neue Schwerpunkte ge- 
setzt werden. Dazu sollten z. B. Elemente wie Feedback-Fahrten, Fahrsicherheitstrainings oder Gruppengespräche gehören.

\section{(2) Fahrlehrerschaft}

Die Ansprüche an die Kompetenzen der Fahrlehrer haben sich u.a. durch die GDE-Matrix verändert. Es ist schwer vorstellbar, dass die heutige Fahrlehrerschaft allein durch ihre Ausbildung in die Lage versetzt werden kann, die gesetzlichen Aufgaben und die Ziele der GDE-Matrix im Rahmen der Fahrschulausbildung zu erfüllen.

- Die Eingangsvoraussetzungen für den Beruf des Fahrlehrers sollten sich an den Zielen der Fahrausbildung orientieren. Denkbar ist z.B. eine Vorbildung in Pädagogik/Psychologie, mindestens ein Realschulabschluss, fünf Jahre unfallfreie und nachweisbar kontinuierliche Verkehrsteilnahme oder auch ein Mindestalter von 25 Jahren (das damit zumindest formal oberhalb des Hochrisikoalters von 18 bis 24 Jahre läge). Die heutigen Voraussetzungen lassen hier noch sehr viel Spielraum für zielführende Verbesserungen.

- Die Ausbildungsdauer für Fahrlehrer sollte bei gleichzeitiger Qualitätserhöhung verlängert werden. Innerhalb von gut neun Monaten kann Wissen vermittelt werden, aber es darf bezweifelt werden, ob auch nur ein annähernd ausreichendes Maß an pädagogisch/didaktischem Know-how vermittelt und gefestigt werden kann. Auch für die Ausbildung zum Fahrlehrer sollte über eine modulare Konzeption nachgedacht werden, eng verzahnt mit Feedback- und Coachingelementen.

- Dauer und Inhalte obligatorischer Fortbildungen für Fahrlehrer sollten an die neuen Herausforderungen angepasst werden.

\section{(3) Verkehrspädagogik/Verkehrspsychologie}

Sowohl in der Fahrschülerausbildung als auch in der Fahrlehrerausbildung können die beiden Geisteswissenschaften Pädagogik und Psychologie zu einer Erhöhung der Qualität bedeutend betragen.

- Insbesondere im Zusammenhang mit einer Mehrphasenausbildung sollte eine Integration dieser Disziplinen erfolgen. Module mit Gruppen-, Coaching- oder Feedbackelementen könnten professionell durchgeführt oder zumindest begleitet werden.

- Auch der ausschließliche Einsatz von Fahrprüfern aus technischen Berufen, zumeist Ingenieuren, sollte diskutiert werden.

\section{(4) Qualitätssicherung}

Auch wenn das Fahrlehrergesetz eine Kontrolle von Fahrschulen im Rhythmus von in der Regel zwei Jahren durch die Erlaubnisbehörde vorschreibt, ist die Qualität derselben offen. Welche Möglichkeiten haben Verwaltungsbeamte, aufgrund ihrer Ausbildung und Erfahrung, eine kompetente Kontrolle durchzuführen? Sind sie tatsächlich in der Lage, die pädagogischen Konzepte und deren Umsetzung zu beurteilen? Heute ist wohl verständlicherweise von einer Überprüfung der Einhaltung der gesetzlichen Formvorschriften auszugehen.

- Es sollte ein standardisiertes Qualitätssicherungssystem bundesweit einheitlich eingeführt werden. Es müsste eine neutrale Begutachtungsstelle geschaffen werden. Inhaltlich könnte sich am System der „Pädagogisch qualifizierten Fahrschulüberwachung PQFÜ“ [5] des Landes Brandenburg orientiert werden. 


\section{(5) Erweiterte Angebote}

Junge Fahranfänger erreicht man nicht nur in der Fahrschule, sondern auch in den allgemeinbildenden und Berufsschulen. Will man eine Sensibilisierung für sicheres Fahrverhalten erreichen, bietet es sich an, auch außerhalb der Fahrschule neue Anspracheformate zu entwickeln oder erprobte zu verbreiten.

- Als erfolgreich evaluiert wurde ein Pilotversuch in Niedersachsen, in dem Schulen und Fahrschulen eng zusammenarbeiteten [6]. Jugendliche Führerscheinbewerber nahmen parallel zum Fahrschulunterricht an einer schulischen Arbeitsgemeinschaft mit einem Umfang von 14 Doppelstunden teil (Führerschein-AG). Die Führerschein-AG stieß bei allen Beteiligten auf eine hohe Akzeptanz und trug dazu bei, das Jugendlichkeitsrisiko zu senken. Nachweislich beeinflusste die aktive Teilnahme an der AG im Vergleich zur Kontrollgruppe den Fahrstil positiv, Verkehrsregeln wurden konsequenter eingehalten und die Anzahl kritischer Situationen bei der Verkehrsteilnahme reduziert. Ein Einfluss auf das Anfängerrisiko konnte nicht nachgewiesen werden.

\section{(6) Örtlicher Alltagsbezug}

Junge Fahrer verunglücken zumeist auf den für sie typischen Wegen, auf der Fahrt zur Arbeit/Ausbildungsstätte, zu Freizeiteinrichtungen oder zu Freunden. Sie machen ihre Fahrausbildung in aller Regel am Wohnort. Daraus ergibt sich, dass sich Fahranfänger überwiegend in einem abgrenzbaren Bereich rund um ihr Zuhause im Verkehr bewegen.

- Diese Information sollte Eingang in die praktische Fahrausbildung finden. Fahrlehrer könnten ihre Schüler nach ihren bevorzugten Wegen fragen und diese verstärkt in die Fahrstunden einbinden.
- Das Pilotprojekt „RegioProtect 21“ [7] in Brandenburg hat zum Ziel, die für Fahranfänger nachweislich gefährlichen Straßenabschnitten in die Fahrstunden, aber auch in den theoretischen Unterricht, zu integrieren. Auf Grundlage des realen Unfallgeschehens, das der Polizei bekannt ist, werden Streckenabschnitte, auf denen Unfälle mit jungen Fahrern passiert sind, identifiziert und können so mit dem Fahrschüler befahren, geübt und reflektiert werden. Das Projekt ist derzeit noch nicht abgeschlossen.

\section{Fazit}

Das Unfallgeschehen junger Fahranfänger macht deutlich, dass weiterhin Anstrengungen notwendig sind, um junge Menschen in den ersten Jahren ihrer Automobilität zu unterstützen.

Eine verbesserte Fahrausbildung kann hier einen Beitrag leisten. Sie sollte darauf ausgelegt sein, dass

- alle Führerscheinneulinge bis zur vollen selbstständigen Teilnahme am Verkehr mehr Zeit bekommen, unter geschützten Bedingungen Erfahrungen zu sammeln,

- der Lernprozess fachlich kompetent begleitet wird,

- die Bedeutung verkehrssicheren Verhaltens von den Jugendlichen in einen Bezug zum eigenen Alltag gesetzt werden kann.

Dabei kann es nicht darum gehen, die Anzahl von Fahrstunden zu erhöhen, sondern vielmehr um eine Modifizierung der Fahrlehrerund Fahrschülerausbildung insgesamt. Eine veränderte Konzeption der Fahrausbildung darf aber nicht automatisch zu einer Kostensteigerung für die Fahranwärter führen. Daher gilt es, die Ausbildungselemente auch unter dem Gesichtspunkt der Kostenneutralität zu entwickeln. 


\section{Literatur}

[1] Statistisches Bundesamt (Destatis): Fachserie 8, Reihe 7 „Verkehrsunfälle, Wiesbaden 2011

[2] Müller, D.: Optimierumg der praktischen Fahrausbildung, unveröffentlichter Schlussbericht, Unfallforschung der Versicherer, Berlin 2011

[3] EU-Projekt GADGET - Guarding Automobile Drivers through Guidance Education and Technology, Final Report, Vienna 1999

[4] GDE-Matrix nach Hatakka, Keskinen, Glad, Gregersen, Hernetkoski; 2002

[5] Hoffmann, L.: Das System der „Pädagogisch qualifizierten Fahrschulüberwachung (POPÜ)“, Potsdam 2008

[6] Integratives Konzept zur Senkung der Unfallrate junger Fahrerinnen und Fahrer, Berichte der Bundesanstalt für Straßenwesen, Heft M 170, Bergisch-Gladbach 2005

[7] Informationen auf der Projekt-Homepage: http://www.regio-protect-21.de/ 


\section{GDV}

DIE DEUTSCHEN VERSICHERER

\section{Gesamtverband der Deutschen Versicherungswirtschaft e.V.}

Wilhelmstraße 43/43G, 10117 Berlin

Postfach 0802 64, 10002 Berlin

Tel.: 030/2020 - 50 00, Fax: 030/2020-6000

www.gdv.de, www.udv.de 